\title{
A Review on Tumor-Treating Fields (TTFields) Clinical Implications Inferred from Computational Modeling
}

Wenger, Cornelia; Miranda, Pedro C.; Salvador, Ricardo; Thielscher, Axel; Bomzon, Zeev; Giladi, Moshe; Mrugala, Maclej M.; Korshoej, Anders R.

Published in:

I E E E Reviews in Biomedical Engineering

Link to article, DOI:

10.1109/RBME.2017.2765282

Publication date:

2018

Document Version

Publisher's PDF, also known as Version of record

Link back to DTU Orbit

Citation (APA):

Wenger, C., Miranda, P. C., Salvador, R., Thielscher, A., Bomzon, Z., Giladi, M., Mrugala, M. M., \& Korshoej, A. R. (2018). A Review on Tumor-Treating Fields (TTFields): Clinical Implications Inferred from Computational Modeling. I E E E Reviews in Biomedical Engineering, 11, 195-207.

https://doi.org/10.1109/RBME.2017.2765282

\section{General rights}

Copyright and moral rights for the publications made accessible in the public portal are retained by the authors and/or other copyright owners and it is a condition of accessing publications that users recognise and abide by the legal requirements associated with these rights.

- Users may download and print one copy of any publication from the public portal for the purpose of private study or research.

- You may not further distribute the material or use it for any profit-making activity or commercial gain

- You may freely distribute the URL identifying the publication in the public portal 


\title{
A Review on Tumor-Treating Fields (TTFields): Clinical Implications Inferred From Computational Modeling
}

\author{
Cornelia Wenger $^{(\mathbb{1})}$, Pedro C. Miranda ${ }^{(\mathbb{0}}$, Ricardo Salvador ${ }^{(0)}$, Axel Thielscher, Zeev Bomzon, \\ Moshe Giladi, Maciej M. Mrugala, and Anders R. Korshoej
}

\begin{abstract}
Tumor-treating fields (TTFields) are a cancer treatment modality that uses alternating electric fields of intermediate frequency $(\sim 100-500 \mathrm{kHz})$ and low intensity (1-3 V/cm) to disrupt cell division. TTFields are delivered by transducer arrays placed on the skin close to the tumor and act regionally and noninvasively to inhibit tumor growth. TTFields therapy is U.S. Food and Drug Administration approved for the treatment of glioblastoma multiforme, the most common and aggressive primary human brain cancer. Clinical trials testing the safety and efficacy of TTFields for other solid tumor types are underway. The objective of this paper is to review computational approaches used to characterize TTFields. The review covers studies of the macroscopic spatial distribution of TTFields generated in the human head, and of the microscopic field distribution in tumor cells. In addition, preclinical and clinical findings related to TTFields and principles of its operation are summarized. Particular emphasis is put on outlining the potential clinical value inferred from computational modeling.
\end{abstract}

Index Terms-Cancer treatment, computational modeling and simulation, finite element models, tumor-treating fields (TTFields).

Manuscript received May 12, 2017; revised August 14, 2017; accepted September 21, 2017. Date of publication February 13, 2018; date of current version July 24, 2018. (Corresponding author: Cornelia Wenger.)

C. Wenger is with the Novocure GmbH, Root D 6039, Switzerland (e-mail: CWenger@novocure.com).

P. C. Miranda is with the Institute of Biophysics and Biomedical Engineering, Faculty of Science, University of Lisbon, Lisbon 1794-016, Portugal (e-mail: pcmiranda @fc.ul.pt).

R. Salvador is with the Institute of Biophysics and Biomedical Engineering, Faculty of Science, University of Lisbon, Lisbon 1794-016, Portugal, and also with the Neuroelectrics, Barcelona 08035, Spain (e-mail: rnsalvador@fc.ul.pt).

A. Thielscher is with the Danish Research Centre for Magnetic Resonance, Copenhagen University Hospital Hvidovre, Hvidovre 2650, Denmark, with the Max Planck Institute for Biological Cybernetics, Tübingen, Germany, and also with Biomedical Engineering, DTU Elektro, Technical University of Denmark, Kongens Lyngby 2800, Denmark (e-mail: axthi@elektro.dtu.dk).

Z. Bomzon and M. Giladi are with the Novocure Ltd., Haifa 34404 , Israel (e-mail: zbomzon@novocure.com; mgiladi@novocure.com).

M. M. Mrugala is with the Department of Neurology, University of Washington, Seattle, WA 98195, USA (email: mmrugala@uw.edu).

A. R. Korshoej is with the Department of Neurosurgery, Aarhus University Hospital, Aarhus 8000, Denmark, and also with the Department of Clinical Medicine, Aarhus University, Aarhus 8000, Denmark (email: andekors@rm.dk).

Digital Object Identifier 10.1109/RBME.2017.2765282

\section{INTRODUCTION}

\section{A. Electric Field Interactions With Biological Tissue}

T IS WELL established that electric fields (EFs) affect cellular function. Direct current or low-frequency alternating fields $(<1 \mathrm{kHz})$ affect the polarization of cell membranes [1] and induce excitatory or inhibitory effects on electrically excitable tissue. Thus, medical applications utilizing low-frequency fields target a multitude of diseases in a range of tissues, including neural and muscle tissue [1]. High-frequency fields (MHz range) do not induce membrane polarization but rather cause fast oscillation of polar molecules (friction) [2] leading to tissue heating. Therefore, high-frequency electromagnetic fields are used for applications, such as diathermy and tissue and tumor ablation [3]. Until recently the intermediate frequency range of several hundred $\mathrm{kHz}$ had not been considered for medical applications, since currents in this range alternate too fast to stimulate nerves or muscles and induce only minute heating through ohmic and dielectric loss [4]. However, since the early 2000s, several studies have shown that intermediate frequency fields disrupt cell division in cancer cells [5], [6]. These observations have led to the development of tumor-treating fields (TTFields) [5], [6], a physical modality for treating cancer.

\section{B. TTFields: Preclinical Observations and Mechanisms of Action}

TTFields are alternating EFs in the frequency range of 100 $500 \mathrm{kHz}$ and intensities typically in the range $1-3 \mathrm{~V} / \mathrm{cm} \mathrm{[5]} \mathrm{that}$ exert an antimitotic effect on cells. TTFields have been studied in vitro using several preclinical laboratory research systems that have been developed for this purpose. These systems include the Inovitro system (Novocure Ltd.) [7], which consists of a TTFields generator connected to a set of ceramic petri dishes, microfluidic devices [8], and wire-based devices [5], [9], [10]. Using these types of devices, researchers have shown that exposing cells to TTFields leads to prolonged mitosis, the formation of abnormal mitotic figures, and mitotic cell death [5], [11], [12]. TTFields also induce violent membrane blebbing during telophase, which in turn leads to the formation of abnormal daughter cells and induction of cell death in the following interphase [5], [13]. The antimitotic effect of TTFields is syn- 
ergistic with the effects of chemotherapeutic agents [14]-[16]. When combined with TTFields, chemotherapeutic agents delivered at doses well below the therapeutic threshold lead to complete cell cycle arrest [14]. Pavesi et al. showed that TTFields application leads to reduced proliferation of breast cancer cells, whilst leaving normal human endothelial cells largely unaffected [8]. Recent studies have also demonstrated that TTFields can inhibit cell migration [9] and DNA damage repair [10], [17].

TTFields effect is intensity dependent. Cell growth rate decreases as field intensity increases. For most cell lines, growth rates begin to decrease when the field intensity exceeds a threshold of about $1 \mathrm{~V} / \mathrm{cm}$, and growth is completely arrested when field intensity exceeds about $2.5 \mathrm{~V} / \mathrm{cm}$ [5], [6]. The effect is also frequency dependent, and each cell line has an optimum frequency, at which the inhibitory effect on cell growth is most significant [5], [6]. For glioma cells, the optimal frequency is $200 \mathrm{kHz}$ [5], [6]. In addition, cells dividing parallel to the field are more likely to be affected by the field than cells dividing in other directions [6], and periodically switching the field between two orthogonal field directions was shown to be $20 \%$ more effective compared to applying TTFields in a single direction [6]. Finally, cells with a short doubling time are more susceptible to the effect TTFields [12], likely due to the higher frequency of mitotic events in a given time.

It has been proposed that TTFields exert their effect by causing alignment of proteins with large dipole moments with the EF [5], [6], thereby disrupting structures and inhibiting protein polymerization. Indeed, two proteins with large dipole moments, tubulin dimers (1740 Debye [18]) and septin (2711 Debye [13]) are affected by TTFields. Studies show that TTFields disrupt microtubule (MT) polymerization, preventing proper chromosome segregation during mitosis [12], and that under the influence of TTFields, septin, which serves as scaffold for the actin myosin ring closing the cytokinetic furrow, fails to localize to the cell mid-zone leading to ectopic blebbing and abnormal mitosis [13].

To explain the effect of TTFields, it has also been suggested that when TTFields are applied to cells during telophase, the hourglass shape of the dividing cell causes the EF within the cell to become highly nonuniform with higher field intensities close to the narrow furrow region. Such field inhomogeneity results in dielectrophoretic (DEP) forces [19] possibly leading to irregular aggregation of polarizable particles, thereby disrupting cell division [4], [5].

These two theories were recently evaluated in a theoretical analysis by Tuszyński et al. [20]. According to this analysis, the DEP forces that develop during telophase are indeed strong enough to interfere with mitosis. However, the forces and torques that the EF exerts on the intrinsic dipoles of tubulin are too small to have a significant effect on tubulin alignment. This analysis demonstrates that although the biological effects of TTFields on cells are well documented, there is still a need for in-depth biophysical studies to elucidate the physical mechanisms by which TTFields exert their biological effects [21].

\section{TTFields: Clinical Trial and Clinical Settings}

The clinical application of TTFields has been tested in animal models [5]-[7], [11], [22], [23] and several clinical trials, the first of which demonstrated that exposure to TTFields led to local regression of skin metastases from breast cancer and melanoma [24]. A pilot trial with patients suffering from glioblastoma multiforme (GBM) showed that treatment with TTFields is feasible and well tolerated without causing serious adverse effects [25]. A subsequent phase III clinical trial (EF-11) compared the efficacy of TTFields to the physician's best choice of chemotherapy in recurrent GBM patients [26]. This trial showed comparable efficacy between the two arms with a better quality of life and less toxicity for TTFields patients [26] leading to the U.S. Food and Drug Administration (FDA) approving TTFields for the treatment of recurrent GBM in 2011. The subsequent EF-14 trial showed that treating newly diagnosed GBM patients with a combination of TTFields and temozolomide led to a 5 month increase in median overall survival (hazard ratio $0.65, p<0.00005$ ) relative to patients treated with temozolomide alone [27]-[29]. TTFields combined with temozolomide was approved by the FDA for the treatment of newly diagnosed GBM in October 2015.

Clinical trials testing feasibility and safety and/or efficacy of applying TTFields to nonsmall cell lung cancer (Lunar [30], [31]), pancreatic (Panova-NCT01971281 [32]), ovarian cancer (Innovate-NCT02244502), mesothelioma (StellarNCT02397928), and brain metastasis (Metis-NCT02831959, Comet-NCT01755624) have also been initiated.

When treating GBM, TTFields are delivered using the Optune system (Novocure Ltd). The system weighs $1.3 \mathrm{~kg}$ and comprises a portable battery powered field generator, which is connected to two pairs of transducer arrays placed on the shaved scalp of the patient. One pair of arrays is placed on the left and right sides of the head (LR array), and the other pair on the anterior and posterior aspects of the head (AP array). Each array consists of nine ceramic disks arranged in a $3 \times 3$ configuration [33]. The device delivers a maximum current of $2000 \mathrm{~mA}$ peakto-peak at a frequency of $200 \mathrm{kHz}$. At any given instance, the current is delivered to only one pair of arrays, and the direction of the field is switched between the pairs of arrays once per second. Patients are treated outside the hospital environment and advised to wear the device for a minimum of $18 \mathrm{~h}$ per day to ensure effective treatment [33]. The transducer arrays are disposable and replaced every few days [33], [34]. The same principles apply to treatment of other body regions, with the frequency of the field and the geometry and positioning of the transducer arrays adapted for each application. For instance, in clinical trials, lung cancer is treated using the NovoTTF-100L device, which delivers TTFields at $150 \mathrm{kHz}$ and is configured with larger transducer arrays than the Optune device [31], [35].

The placement of Optune transducer arrays on the patient's scalp (referred to as layout) is determined using the NovoTAL system [36], [37]. NovoTAL uses measurements of the head and tumor obtained from axial and coronal MR images of the patient to derive array layouts that maximize the field delivery to the tumor. Treatment planning with NovoTAL was defined 
as part of the protocol for the EF-14 trial, and is standard when initiating TTFields treatment [36]. Computational studies have shown increased EF delivery to the diseased region [38], [39] when arrays are adapted to the individual tumor location.

\section{Article Motivation and Purpose}

Many factors are known to influence the outcome of GBM treatment. These factors include age, Karnofsky performance status, the extent of the resection, and morphological, histological, and genetic characteristics, such as isocitrate dehydrogenase (IDH) and $\mathrm{O}(6)$-methylguanine-DNA methyltransferase (MGMT) tumor mutation status [40], [41]. When treating with TTFields, it is reasonable to assume that in addition to the factors mentioned above [21], the distribution and intensity of the induced $\mathrm{EF}$ in the brain and tumor also influence treatment outcome. The field distribution and intensity may vary significantly between individuals depending on the size, shape, and electrical properties of the head, brain, and tumor. It would be highly desirable to measure the field intensities in the regions of interest. However, presently this task remains challenging. Hence, numerical simulations are the best available tool for evaluating TTFields distributions within patients.

In this paper, we will review computational studies analyzing the application of TTFields. We will show how modeling TTFields at the cellular level can be used to investigate the biophysical basis for TTFields mechanisms of action, and how realistic human head models can be used to investigate the connection between EF distribution and efficacy and safety of TTFields treatment. We will discuss how, in the future, these models could be employed to study treatment response, predict progression patterns, conduct retrospective outcome analysis, enable prospective personalized treatment planning, and possibly help to select suitable candidates for therapy.

\section{Computational Models of TTFieldS}

Whether simulating TTFields delivery at the cellular scale (see Section II-A) or at the full head scale (see Section II-B), the EF distribution is approximated by applying volume conductor models [1] to numerically solve derivatives of Maxwell's equations of electrodynamics with appropriate boundary conditions (BCs). For TTFields, the electromagnetic wavelength is much larger than the size of the models, and therefore the electroquasistatic approximation of Maxwell's equations may be applied [42]. Thus, the electric potential $V$ can be found by solving the Laplace equation, $\nabla \cdot \tilde{\sigma} \nabla V=0$. Here, $\tilde{\sigma}=\sigma+i \omega \varepsilon$ is the complex conductivity, $\varepsilon$ is the permittivity, $\sigma$ the electrical conductivity, and $i$ the imaginary unit, and $\omega=2 \pi f$ the angular frequency. The numerical task of solving the Laplace equation can be achieved using, for example, the finite element (FE) method. Table I illustrates the similarities and differences between cellular scale and full head scale models for studying TTFields.

\section{A. Cellular Level Modeling}

1) Materials and Methods: Glioma cells have been found to round up during mitosis to a sphere of approximately $5500 \mathrm{fL}$
TABLE I

COMPUTATIONAL MOdELING OF TT FIELDS APPLICATION

\begin{tabular}{|c|c|c|}
\hline & Cellular level model & Human head model \\
\hline purpose & $\begin{array}{l}\text { study of TTFields } \\
\text { mechanism of action }\end{array}$ & $\begin{array}{l}\text { study of TTFields clinical } \\
\text { application }\end{array}$ \\
\hline related device & Inovitro & Optune \\
\hline model creation & $\begin{array}{l}\text { simple geometric } \\
\text { objects }\end{array}$ & MRI tissue segmentation \\
\hline model physics & $\begin{array}{l}\text { electro-quasistatic } \\
\text { approximation of } \\
\text { Maxwell's equations }\end{array}$ & $\begin{array}{l}\text { electro-quasistatic } \\
\text { approximation of Maxwell's } \\
\text { equations }\end{array}$ \\
\hline model result & $\begin{array}{l}\text { EF distribution in the } \\
\text { extracellular space, } \\
\text { the cell membrane, } \\
\text { and cytosol }\end{array}$ & $\begin{array}{l}\text { EF distribution in the head } \\
\text { and tumor tissues }\end{array}$ \\
\hline
\end{tabular}

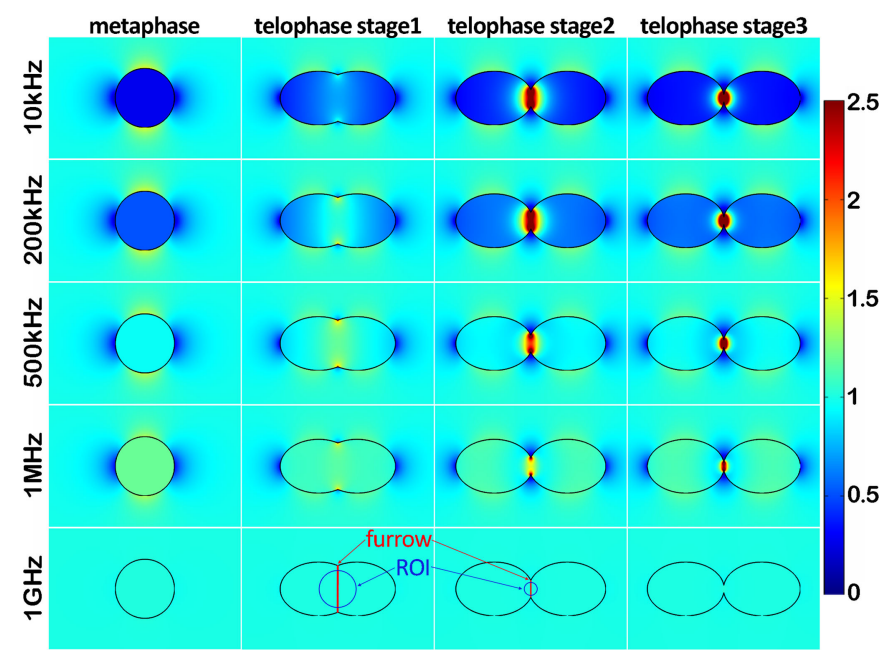

Fig. 1. $E_{i}$ distribution in the metaphase cell (column1) and three stages of telophase (columns 2-4) for varying frequency (rows) of the applied $\mathrm{EF}$ from left to right with an intensity of $1 \mathrm{~V} / \mathrm{cm}$. The color scale is fixed for all panels from $0-2.5 \mathrm{~V} / \mathrm{cm}$. The bottom panel illustrates the furrow length (red line) and spherical ROI (blue circle) in the telophase cells.

(radius of approximately $11 \mu \mathrm{m}$ ) [43]. According to images of the division process [43], as the cell proceeds into telophase elliptical cell shapes with equal size become more appropriate to characterize the process in which the daughter cells form.

To reflect these geometries, studies simulating TTFields application to single cells [20], [44]-[46] assumed a spherical cell with a default radius of $10 \mu \mathrm{m}$ during metaphase. During telophase and cytokinesis, elliptical sister cells with a default major radius of $10 \mu \mathrm{m}$ and a minor radius of $7 \mu \mathrm{m}$ were considered. Three different stages of telophase were modeled by decreasing the length of the incision plane between the sister cells (furrow length) as division progressed (see red line in the bottom panel of Fig. 1).

The models comprised of three domains: the extracellular space, the cytosol, and the membrane, which differ in their dielectric properties. The standard values assigned to these tissue types were $\sigma_{i}=0.3 \mathrm{~S} / \mathrm{m}, \sigma_{e}=1.2 \mathrm{~S} / \mathrm{m}, \sigma_{m}=3 \mathrm{e}-7 \mathrm{~S} / \mathrm{m}$, $\varepsilon_{i}=72.3, \varepsilon_{e}=72.3, \varepsilon_{m}=5$. These values were adapted from computational studies conducting similar investigations [47][56]. Since a large range of properties are expected within the 


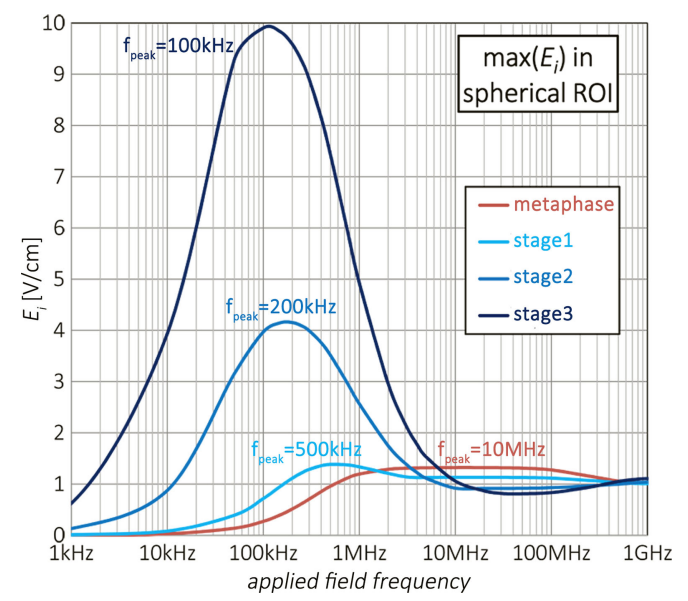

Fig. 2. Maximum $E_{i}$ as a function of frequency for four different cell cycle stages. The values are measured in central ROls with a radius of $80 \%$ of the furrow length.

population of glial cells [46], different ranges of these parameters were tested to investigate how the dielectric properties of cells influence their response to TTFields (see Section II-A-2). The computational studies [20], [44]-[46] were carried out using the Electric Currents Interface of the ac/dc module of Comsol Multiphysics (www.comsol.com). The thin membrane was numerically represented by the contact impedance $\mathrm{BC}$ available in Comsol.

In order to apply a homogenous $\mathrm{EF}$, the $\mathrm{BCs}$ on two opposite sides were chosen as terminals of the voltage type where the others were electrically insulated, representing an ideal parallel plate electrode setup. The default applied voltages of opposite signs were chosen to produce standard field intensity of $1 \mathrm{~V} / \mathrm{cm}$, which corresponds to the minimum activity threshold of TTFields intensity. The computational model was validated by comparing numerical results for a spherical cell to the analytical description of the transmembrane voltage of a spherical cell with radius $r$ (data not shown) [52].

\section{2) Results}

a) Effect of field frequency: The distribution of the EF intensity in and around a cell with standard geometric and dielectric properties (see Section II-A-1) is plotted in Fig. 1 for selected frequencies (rows) at different stages of mitosis (columns). For a round cell during metaphase, the EF distribution inside the cell is uniform with only small perturbations close to the membrane. The intracellular field intensity, $E_{i}$ almost equals zero for frequencies $<10 \mathrm{kHz}$ (left top in Figs. 1 and 2). For increasing frequency, $E_{i}$ gradually increases and finally equals the applied field intensity for frequencies $>1 \mathrm{GHz}$ as predicted by Figs. 1 and 2 and discussed in detail elsewhere [45], [46].

During telophase, the low-frequency fields do not penetrate the membrane and $E_{i}$ is close to zero (column 2 in Figs. 1 and 2). However, as frequencies increase into the $\mathrm{kHz}$ range, a nonuniform field develops within the cytosol with higher field intensities found close to the furrow between the dividing cells (see columns 2-4 in Fig. 1). The most pronounced and spatially confined $\mathrm{EF}$ at the furrow is found for a stage 3 cell (see column 4 in Fig. 1). As the frequency of the field increases, the nonuniformity close to the furrow decreases and the field intensity throughout the cell increases. Thus, $E_{i}$ peaks between $100-500 \mathrm{kHz}$ during telophase, with lower peak frequencies observed for later stages of cell division (see Fig. 2).

For this review article, we evaluated the maximum of $E_{i}$ in a spherical region of interest (ROI-blue circles in bottom panel of Fig. 1) located at the center of each cell, with a diameter that corresponds to $80 \%$ of the furrow length (see Fig. 2). The figure shows that the maximum value of $E_{i}$ increases as the cell progresses through cell division and that its value can exceed the external field strength of $1 \mathrm{~V} / \mathrm{cm}$. Second, the frequency for which the maximum $E_{i}$ occurs decreases as the cell division progresses (see Fig. 2). As a result of the field inhomogeneity inside the dividing cell, the gradient of the $\mathrm{EF}$ becomes nonzero. Hence, the DEP force component defined as $\nabla\left|\boldsymbol{E}_{i}\right|^{2}$ [57], [58] also takes on nonzero values, increasing to maximal values during later stages of telophase with highest values for stage3.

b) Effect of cell size: Subsequently, we investigated how the geometric and dielectric properties of the cell influence the EF distribution. Fig. 3 shows the maximum of $E_{i}$ as a function of frequency for cell radii $r=\{4,10,15,20 \mu \mathrm{m}\}$. During metaphase the biggest cell with $r=20 \mu \mathrm{m}$ has the highest $E_{i}$ for all field frequencies, (see Fig. 3(a)), and the peak frequency at which the highest $E_{i}$ is found in a cell $(10 \mathrm{MHz})$ is unaffected by cell radius. During telophase the biggest cell has the highest $E_{i}$ only for low frequencies [see Fig. 3(b)-(d)]. As the cell radius increases, maximum values of $E_{i}$ occur at lower frequencies [see insets of Fig. 3(b)-(d)]. When a constant field frequency of $200 \mathrm{kHz}$ is applied, cell size influences the field exposure inside the cell (see vertical lines in Fig. 3). During metaphase and stage1 [see Fig. 3(a) and (b)], the highest $E_{i}$ is found in the biggest cell and the lowest $E_{i}$ in the smallest cell, whereas for stage 2 and stage 3 the maximum $E_{i}$ is found in cells of smaller size [see Fig. 3(c) and (d)].

It is interesting to note that the differences in the maximal values of $E_{i}$ obtained at different cell sizes are smaller than 3\%, as shown by the similar height of curves in Fig. 3 and the dotted lines in Fig. 4. This might suggest that the effect of TTFields can be maintained if the frequency is tuned to match the cell size. However, the simulations show that the magnitude of the DEP force component $\left.|\nabla| \boldsymbol{E}_{i}\right|^{2} \mid$ is inversely proportional to the cell size, likely leading to less effective exposure for bigger cells (see the solid lines in Fig. 4).

c) Effect of cell's dielectric properties: Previous computational studies predicted that the dielectric properties, $\sigma$ and $\varepsilon$, of the cell are important determinants of the values of $E_{i}$ and $\left.|\nabla| \boldsymbol{E}_{i}\right|^{2} \mid$. The most influential parameters were found to be $\sigma_{i}, \sigma_{m}$, and $\varepsilon_{m}$, details can be found in [45] and [46]. For all parameter variations, peak frequencies varied between $125 \mathrm{kHz}-$ $1 \mathrm{MHz}$ for stage 1, 50-400 kHz for stage2, and 50-250 kHz for stage3. Another consequence of changing the cells dielectric parameters is that for a fixed field frequency of $200 \mathrm{kHz}$, induced values of $E_{i}$ and $\left.|\nabla| \boldsymbol{E}_{i}\right|^{2} \mid$ might differ significantly.

Apart from the cell's intrinsic geometric and dielectric properties, the intensity and direction of the externally applied $\mathrm{EF}$ influence the EF distribution inside and around the cell. The 


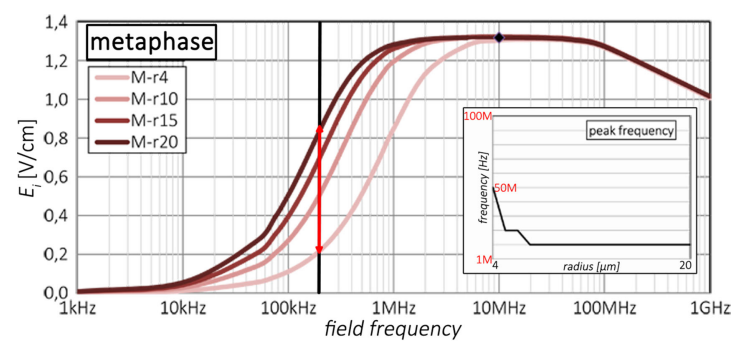

(a)

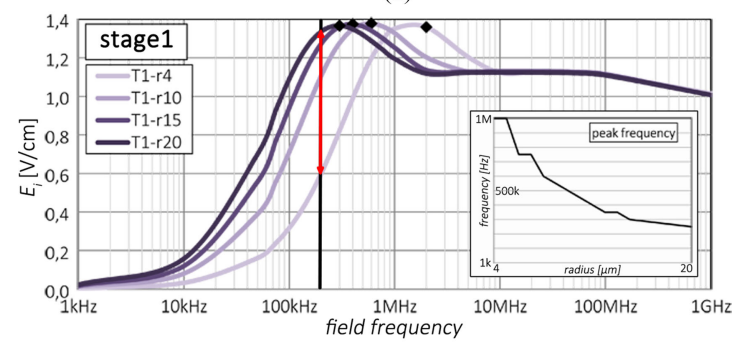

(b)

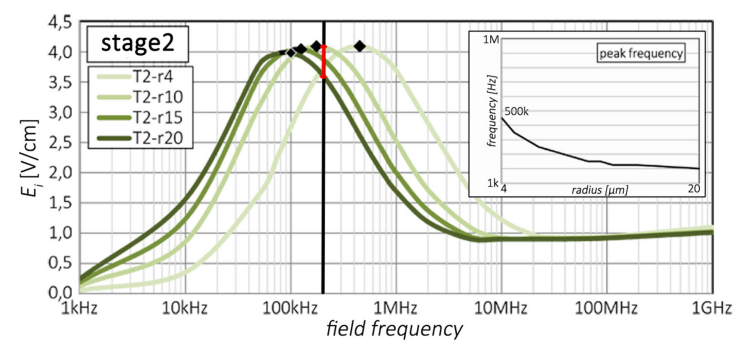

(c)

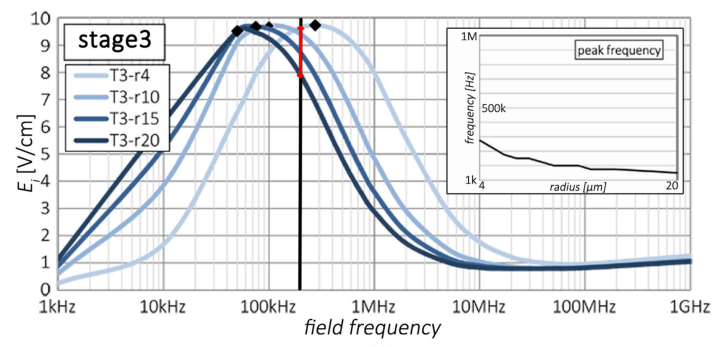

(d)

Fig. 3. Maximum $E_{i}$ as a function of frequency for four selected cell sizes (a) during metaphase and different stages of telophase (b)-(d). The insets in (b)-(d) plot the peak frequency as a function of cell radius, corresponding peaks of the four selected cells are marked as black diamonds on the curves. The vertical line marks $200 \mathrm{kHz}$ and the span of values is emphasized in red.

induced values of $E_{i}$ increase proportionally with the increasing field intensity and the DEP force component exhibits a quadratic growth [44].

d) Effect of cell orientation: The connection between the orientations of dividing cells relative to the field has also been investigated. The field's orientation to the spherical metaphase cell does not change $E_{i}$. The intracellular field remains uniform with parallel EF lines perpendicular to the applied field. But when the cell shape becomes irregular during telophase, the angle between the division axis of the cell and the applied field has significant impact on the intracellular field distribution. The higher the angle between the furrow and the field, the lower the induced maximum $E_{i}$ within the cell. This is true for frequencies below $1 \mathrm{MHz}$, after which the behavior is

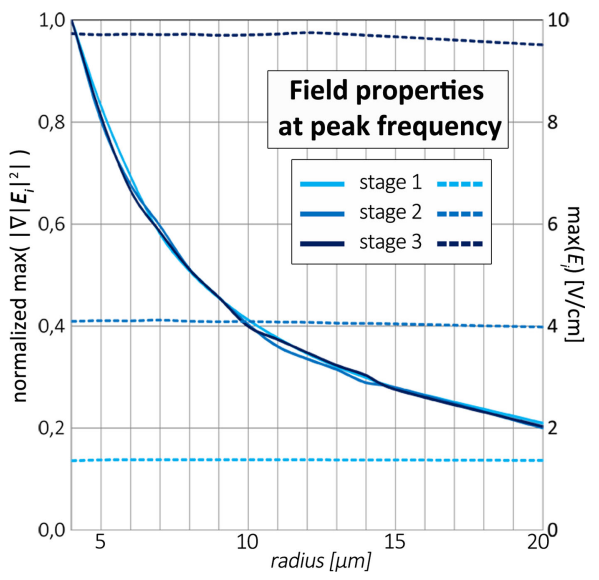

Fig. 4. For each tested cell radius $(r=4-20 \mu \mathrm{m})$, the normalized maximum of $\left.|\nabla| \boldsymbol{E}_{i}\right|^{2} \mid$ is plotted as solid lines (left primary axis) and the maximum of $E_{i}$ as dotted lines (right secondary axis). Values measured in central ROIs.

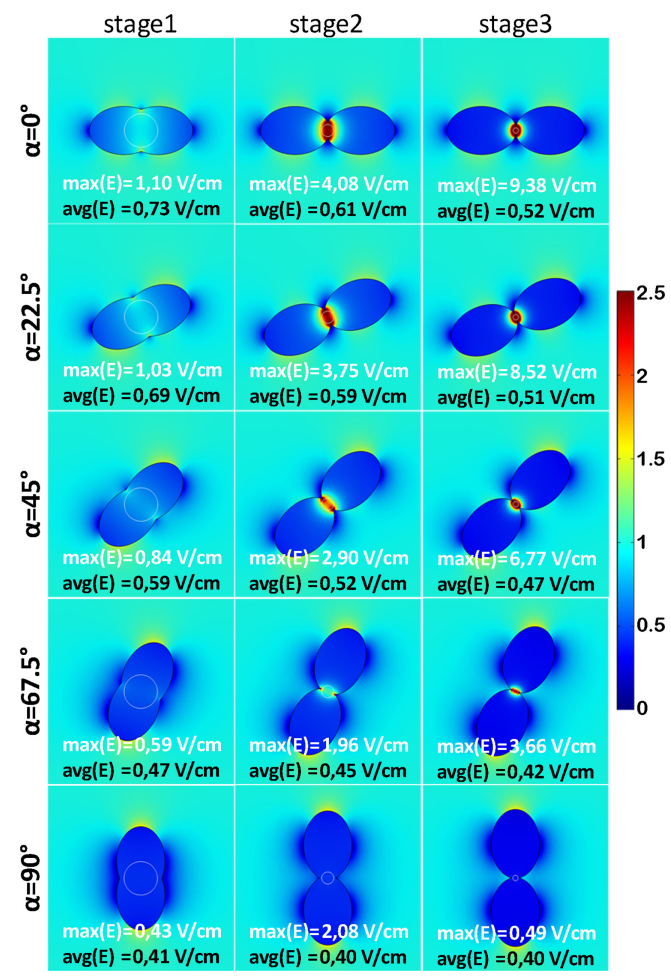

Fig. 5. $E_{i}$ distribution induced in a cell during three stages of telophase (columns) for varying angle between the division axis and the EF (rows). The field has a frequency of $200 \mathrm{kHz}$, an intensity of $1 \mathrm{~V} / \mathrm{cm}$, and it is applied from left to right. The maximum $E_{i}$ in a spherical ROI (white circle) is presented in each panel in white and the corresponding average $E_{i}$ in the whole cell in black.

reversed (results not shown). As example, Fig. 5 plots the field distribution at $200 \mathrm{kHz}$ for the 3 stages (columns) and 5 different angles from $0^{\circ}$ to $90^{\circ}$ (rows). The decreasing maximum values within the spherical ROIs (white circle in Fig. 5) are printed in the panels. Furthermore, the average EF within the whole cells also decreases (see the black values in Fig. 5). In each column, the $E_{i}$ in the highly exposed furrow region diminishes as the angle between division axis and field increases from top to bottom. 
e) Discussion and future directions: The ultimate goal of the computational models presented above is to provide insight into the biophysical mechanisms that govern TTFields, starting with the examination of the connection between the intracellular field distribution and the field parameters (mentioned in Section I-B) that influence treatment efficacy.

Field frequency: Experimental results predict that the efficacy of TTFields is frequency dependent and each cell line has a specific frequency for which the inhibitory effect is highest [5]. Additional in vitro experiments predict that the optimal frequency for the inhibitory effect of TTFields is inversely related to the cell size [5], [6]. The computational models confirm that during cell division, the EF intensity peaks in the range between 100 and $500 \mathrm{kHz}$ with a lower peak frequency for later stages of telophase (see Fig. 2). It should be noted that the target cells are all in different stages of mitosis in a patient, and therefore there is probably no single frequency that is optimal for all of the cells. However, even if one were to be able to synchronize the target cells to be in stage 3 for instance, it might not increase the therapeutic effect of TTFields delivered at a single frequency because of potentially other yet undiscovered biological processes.

The computational models further predict that the cell's geometric (see Fig. 3) and dielectric properties influence the reported peak frequencies. Because different cell lines have different properties, the simulation results might explain the cellline specific optimal frequency. It would be insightful to evaluate and measure the size and dielectric properties of different cell lines [59] and compare them to the computational results. The modeling results show that with increasing the cell size the peak frequencies decrease [see insets Fig. 3(b)-(d)]. The corresponding maximum values of $\left.|\nabla| \boldsymbol{E}_{i}\right|^{2} \mid$ also decreases with the cell size. It is worth noting that experiments showed that cell volume increases in almost all cell lines treated with TTFields [60]. Thus, increasing the cell size during TTFields treatment might be an escape mechanism of the cancer cells, because the intracellular field and DEP component decrease as the cell size changes.

Field Intensity: TTFields' effect increases with intensity [5], and higher field intensities yield smaller cell counts. The computational modeling confirms that increasing the field's intensity also increases the induced intracellular field and DEP component [44]-[46].

Direction of division: In line with experimental results, the computational model predicts decreased intracellular field strengths for cells that are not dividing parallel to the applied field.

When evaluating these results, it is important to keep in mind that the models represent single isolated cells with a homogeneous cytoplasm. Yet, within tissue, cells are generally packed, which could cause local inhomogeneity in the extracellular fields, and could to some extent attenuate field distributions. However, the degree to which TTFields penetrate the cells is determined by the properties of the single cell. Thus, it is unlikely that the observation that the intracellular field and DEP depend on the frequency, intensity, and direction of the applied field will change when considering models of packed cells. Additionally, the cytoplasm is heterogeneous and contains multiple organelles which would increase the nonuniformity of intracellular fields. This could possibly lead to local field gradients and DEP forces even when the cell is rounded during metaphase. Thus, cytoplasmic heterogeneity is expected to magnify the effects of TTFields.

Let us now turn our attention to how the intracellular EF might disrupt mitosis. Endogenous EFs are believed to play a key role during mitosis [61], [62] as supported by experimental evidence for peak electromagnetic activity during mitosis [63], [64] as well as by physical modeling of the electrostatic forces generated by MTs which are at work during mitosis and influence chromosomal motion [65]-[67]. Thus, it is not unlikely that external EFs, such as TTFields could disrupt mitosis. It has been speculated that when dividing cells are exposed to TTFields, the tubulin dimers align with the field rather than with the growing MT axis. However, Tuszyński et al. [20] calculated that the interaction energy of a tubulin dimer with an EF of 1 $\mathrm{V} / \mathrm{cm}$ is too small to affect the dynamics of the dimer. They also proposed that a $1 \mathrm{~V} / \mathrm{cm}$ EF would be insufficient to exert a major influence on MTs and the cytoskeleton due to Debye screening. Furthermore, they showed that the torque an MT experiences in a $1 \mathrm{~V} / \mathrm{cm}$ field is 3 orders of magnitude too low to lead to significant rotation. However, the authors did show that the field nonuniformity in dividing cells could cause substantial DEP forces on tubulin dimers and MTs potentially large enough to disrupt cell division. In addition, they showed that under the influence of TTFields, significant ionic currents could develop along MTs that might disrupt cellular function. In the future it would be useful to create complex cellular models by combining the computational models presented here with electromechanical models of mitotic spindle dynamics [68], [69]. These models have already been adapted to address possible mechanisms of the effects of ultrashort electrical and mechanical pulses on dividing cells [70]. It would also be useful to measure the magnitude of TTFields within the cells. This task is challenging. However, a promising technique that can measure local EF in cell cultures has been reported [71]. In the future, similar techniques might be applicable to experimentally measure the magnitude of TTFields within cells.

\section{B. Realistic Human Head Modeling}

1) Materials and Methods: The creation of realistic human head models is a challenging task, which relies on the segmentation of MRI datasets. Typically, the healthy human head will be divided into tissue types of distinct dielectric properties: the scalp, the skull, the cerebrospinal fluid (CSF), the gray matter (GM) and the white matter (WM). The CSF filled ventricles in the center of the head will also usually be identified. There are many different software packages available to achieve this task in a semiautomated manner.

a) Head model creation: The first model created for TTFields simulation studies [72], [73] originated from T1 and proton density weighted MRIs (http://brainweb.bic.mni. mcgill.ca/brainweb) of the Colin27 dataset. The freely available Brainsuite software was used for segmentation [74]. The second model used a dataset of a young healthy female consisting of T1, T2, and diffusion MRI (dMRI) datasets [75]. The 

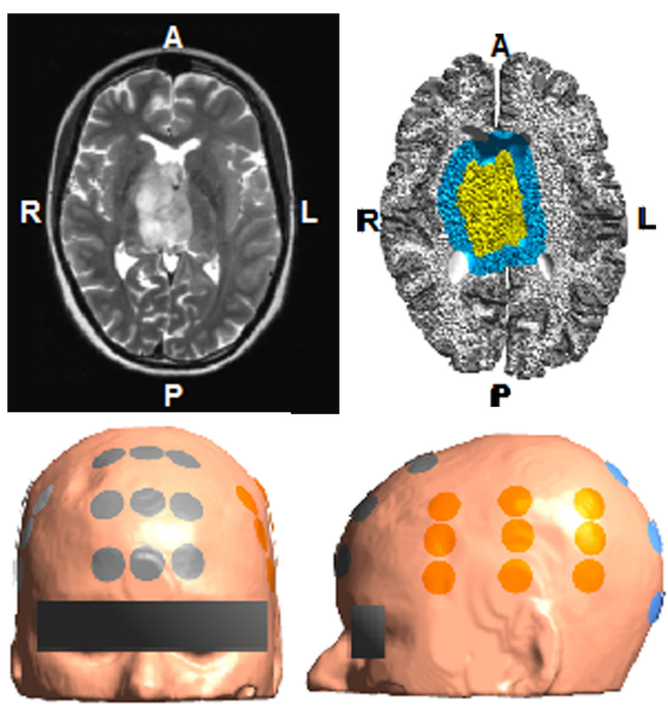

Fig. 6. Example of patient-specific head model based on MRI data. The figure shows an axial T2 MRI image (left) obtained from a 23 year old female patient with deeply seated anaplastic astrocytoma. The three rightmost panels show the head model and tissue segmentation, which was created from the MRI data (T1 and T2) from the same patient. Electrode positioning is evident from the surface reconstruction and electrodes are paired orange/white (LR) and gray/blue (AP).

open-source package SimNibs v1 [76], which uses Freesurfer [77] for segmenting the cortical tissues and FSL [78] for segmenting the outer tissue layers, was adapted for this purpose. Brainsuite was used to create the scalp and skull segmentation of the second model. A recent study [79] has employed a similar approach using the almi5 dataset and the SimNibs v2 software (www.simnibs.org). These models relied on datasets of healthy persons, thus the presence of a tumor was modeled by incorporating virtual lesions consisting of two concentric spheres; the inner sphere represented necrosis surrounded by an enhancing tumor [73], [75], [79]. In [79], 24 tumors were systematically introduced at different positions relative to the active transducerarrays. In [39], three more tumor locations were tested, and also more complex shaped, bigger, more realistic dimensions were assumed.

The first model of a patient with recurrent GBM treated with TTFields therapy was created by segmenting structural images with ScanIP, a commercial software [80], [81]. More recently, head models of a patient with GBM and a patient with anaplastic astrocytoma were created using T1, T2, and dMRI datasets [82] (see Fig. 6). To create these models, T1 and T2 data were initially processed using the mri2mesh algorithm in SimNibs v2 (www.simnibs.org) to produce a preliminary volume mesh. Subsequently, the surface mesh structures and binary tissue masks produced by the algorithm were edited manually using custom code based on meshfix [83], FSL, and Freesurfer algorithms. This was done in order to accurately reproduce the patient's anatomy, particularly in the tumor region and its immediate surroundings.

Following segmentation, the transducer arrays have to be modeled. The studies by Wenger et al. used the 3-matic package of the Mimics v.14 software (www.materialise.com) for this task. The transducers were modeled as $1 \mathrm{~mm}$ high cylinders with a radius of $9 \mathrm{~mm}$ and the gel layer was represented by cylinders (0.5-2 mm height) of $10 \mathrm{~mm}$ radius. For head models created by Korshoej et al., this step was performed automatically with a custom-written MATLAB code (www.mathworks.com). In order to complete the model for FE calculation, the final volume mesh has to be created. For models of Wenger et al., this step was performed with Mimics, while open-source tools in SimNibs, Gmsh [84], and meshfix [83] were employed for the patient models.

b) Finite element calculation of the EF distribution: In order to solve the Laplace equation, the virtual tumor studies used the Electric Currents physics of the ac/dc package of Comsol (www.comsol.com). Because of the complexity of the model, the solver had to be chosen carefully to obtain convergence (GMRES with SOR preconditioner). For models by Korshoej et al., FE calculations were performed with SimNibs using GetDP with an implemented Galerkin method [85] and residuals for the conjugate gradient solver required to be $<10^{-9}$.

To simulate delivery of TTFields to the models, suitable BCs have to be applied. Typically, continuity of the normal component of the current density at all interior boundaries and electric insulation at the external boundaries are considered. In both the virtual tumor models and the patient-based models, the external bases of the active transducer arrays were assigned floating potential BCs that set the potential at that boundary so that the integral of the normal component of the current density was 900 $\mathrm{mA}$ per transducer array, summing up to $1800 \mathrm{~mA}$ peak-to-peak for an active pair of transducer arrays.

To complete the model, the dielectric properties of the different tissue types have to be defined. Isotropic values of electrical conductivity and relative permittivity of different tissue types are listed in the corresponding articles [73], [75], [82]. Studies of related techniques showed that incorporating the anisotropic nature of electric conductivity within the WM significantly affects results [86]-[89]. This anisotropy can be captured by processing diffusion tensor imaging (DTI) data which estimates the diffusion tensor for each voxel [90], [91]. For the second virtual tumor model, the raw DTI images were corrected and registered with the FSL diffusion toolbox [92] in order to calculate the principal directions (eigenvectors), principal diffusivities (eigenvalues), and the fractional anisotropy. There exist several ways for the subsequent estimation of the conductivity tensor, as described in detail elsewhere [93].

In models by Wenger et al., the direct mapping (dM) method that assumes a linear relationship between the eigenvalues of the diffusion and conductivity tensors was applied [94], i.e., $\sigma_{v}=s \cdot d_{v}$, where $\sigma_{v}$ and $d_{v}$ are the $v$ th eigenvalues of the conductivity and the diffusion tensors, respectively. Instead of the initially proposed empirical scaling factor $s$ [94] which is not generally valid, an adapted $s$ was considered [95]. Additionally, an adapted volume normalized (vN) mapping method was used, which locally matches the geometric mean of the conductivity tensor's eigenvalues in each voxel to the corresponding isotropic conductivity value (either WM or GM) [96]. In order to incorporate tissue anisotropy into the computational models, 


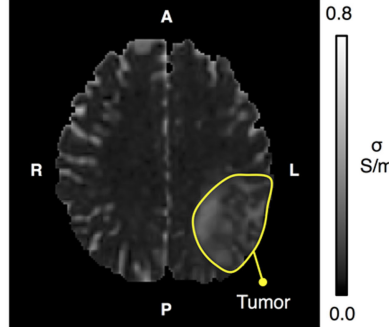

(a)

In vivo

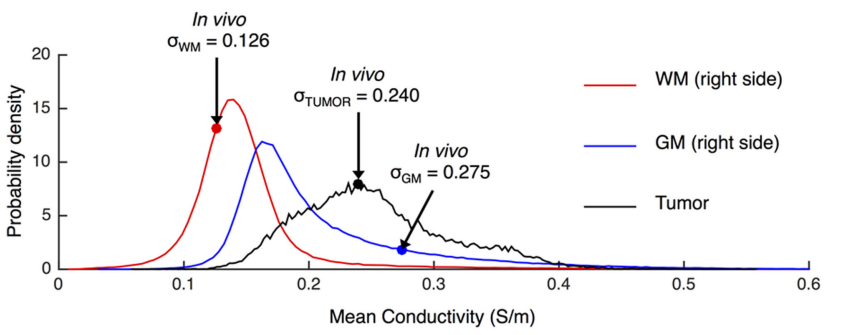

(c)

Fig. 7. Conductivity maps and estimation for patient-based and virtual tumor models. (a) Axial section of dMRI based conductivity map obtained using SimNibs. The map was created using direct linear mapping based on the right GM and WM tissue and then extrapolating the obtained scale factor to obtain a generalized conductivity map covering the whole brain and tumor. (b) The corresponding probability density functions of the conductivity estimates. (c) The fractional anisotropy distribution of the conductivity tensors. As evident from the figures, both distributions in (a) and (b) cover a wide range of values with considerable topographical variability, particularly in the tumor region, which illustrates the potential importance of utilizing patient-specific estimates. It is also notable that the conductivity range and mean values correspond well to in vivo estimates.

the conductivity tensor of each voxel was imported into Comsol for FE computation.

For the patient head models, dMRI processing and conductivity estimation with the DM approach was performed using the $d$ wi2cond algorithm in SimNibs. The scaling factor was obtained by fitting only the nonpathological regions of the GM and WM tissues of the contralateral hemisphere to standardized values, because the technique remains to be firmly validated for tumor tissue. Values in the tumor tissue were obtained by extrapolation using the obtained fit. Fig. 7(a) shows a conductivity map obtained from dMRI data from a patient with left frontoparietal GBM alongside the corresponding probability density functions [see Fig. 7(c)] of the conductivity values. The mean conductivity value in the tumor region was $0.245 \mathrm{~S} / \mathrm{m}$ and the interquartile range was $0.077 \mathrm{~S} / \mathrm{m}$. Thus, the estimated conductivity of the tumor was in the same range as previously reported for gliomas in vivo [97]-[99] and agreed well with the conductivity values used in the virtual tumor models. Fig. 7 also demonstrates the considerable anisotropy (high fractional anisotropy) in the WM tissue, which has been shown to impact the distribution of the estimated field considerably [75]. It is also evident that the tumor region is characterized by considerable topographical variability in both conductivity and fractional anisotropy, which highlights the importance of individualized head modeling and conductivity estimation. After conductivity estimation, the resulting anisotropic conductivity tensors were imported directly into SimNibs for FE calculations.
For the virtual models by Korshoej et al., a similar approach was adopted to produce a conductivity tensor for WM and GM using $d$ wi2cond algorithm. Isotropic conductivities similar to Wenger et al. were used for the remaining tissues, including the tumor and necrosis.

2) Results: The simulation-based studies show that TTFields distribution within the brain is heterogeneous [72], [73], [79], [100] and influenced by the complex tissue interfaces, and especially the differences in dielectric properties of the different tissues. The highest field intensities are found in the scalp and skull close to the active transducer arrays. Although the field in the brain tissue on average decreases with the increasing distance from the transducers, field strengths are not necessarily higher for tumors close to the active arrays. This is mainly due to shunting of current through surrounding less resistive CSF pathways during LR array activation [79]. Deeper regions with higher field intensities (hot spots) were also observed (see Fig. 8), caused by the fact that at a tissue interface the field increases in the lower conductivity medium and decreases in the tissue with higher conductivity. This behavior has also been reported in other noninvasive brain stimulation techniques [101]. In addition, the CSF creates low-resistance pathways causing currents to flow through the sulci, ventricles, and resection cavities toward deeper regions, which in turn creates local field "hot spots" in deeply seated tumors embedded in $\mathrm{WM}$, as well as in tumors located close to the sulcal fundi and near resection borders [79]. Higher EF intensities were found for the LR array and the field distribution appears to be more uniform in the AP setting. Nonetheless, the average EF intensities in the brain were estimated to be very similar in the two settings, $\left|\boldsymbol{E}_{\text {brain }}^{L R}\right|=1.18 \mathrm{~V} / \mathrm{cm}$ and $\left|\boldsymbol{E}_{\text {brain }}^{A P}\right|=1.14 \mathrm{~V} / \mathrm{cm} \mathrm{[75].}$ The AP array configuration delivered higher field intensities to superficial tumors, while the LR configuration delivered higher intensities to deeper tumors [79].

Within the tumor, low field values were found in the necrotic core and higher values with a nonuniform distribution were found in the tumor and peritumoral border zone [73], [79]. Again the directional effects, explained above, were responsible for hotspots created in the tumor, which were far more pronounced in the LR setting for the first tumor location tested [75]. The average EF intensities in the enhancing tumor part depended significantly on the tumor location and ranged between $\left|\boldsymbol{E}_{\text {shell }}^{L R}\right| \approx 1.20-1.65 \mathrm{~V} / \mathrm{cm}$ and $\left|\boldsymbol{E}_{\text {shell }}^{A P}\right| \approx 0.75-1.40 \mathrm{~V} / \mathrm{cm}$ [75], [79].

Another aim of the modeling studies was to clarify to what extent the EF distribution depends on assumptions about the dielectric properties of the tissues, and the extent to which tissue anisotropy influences the field distribution [75], [100]. For the second virtual tumor head model, representation of the conductivity tensor using both the $\mathrm{dM}$ and the vN methods (see Section II-B-1) yielded field distributions that were similar to the field distribution in the isotropic model. The calculated average EF intensities in the brain and tumor showed only minor differences between isotropic and anisotropic models. However, tumor locations in highly anisotropic areas of the WM [79] and possibly also the tumor's intrinsic anisotropy of conductivity can influence the field intensity at the diseased site. Indeed, the field 

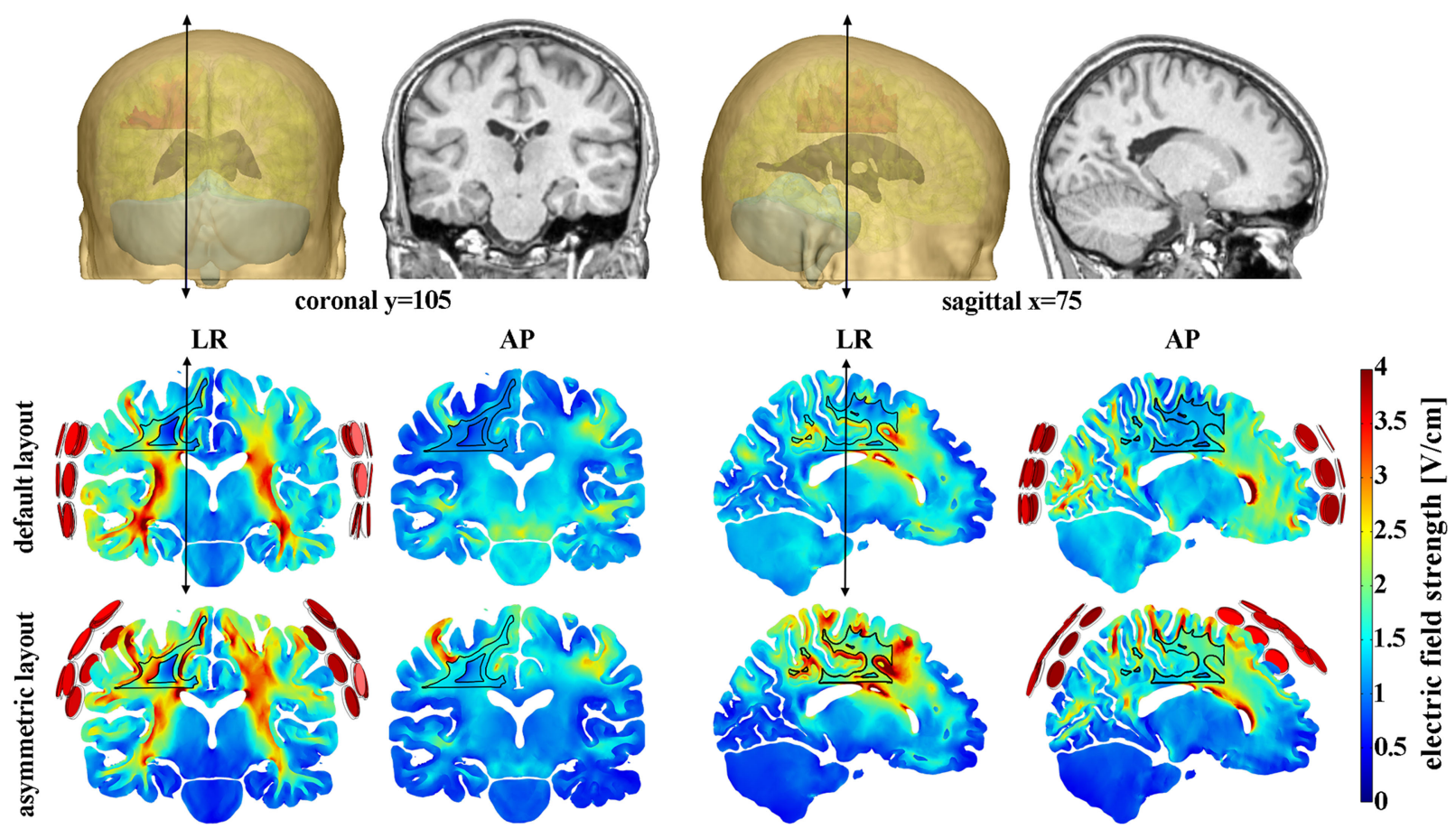

Fig. 8. Distribution of the EF's intensity $(\mathrm{V} / \mathrm{cm})$ is presented in two axial slices through a virtual tumor located frontal in the left hemisphere (contour shown in the left hemisphere, anteriorly). Default (second row) and patient-specific adapted layouts (third row) are presented for LR and AP setups of the Optune device. Corresponding anatomical slices and their position in the head model are presented at the top. Reproduced from [38].

distributions in the patient-based models suggest that the choice of conductivity assignment plays a significant role in determining the field distribution in the regions of pathology. Particularly, the median field intensity is approximately $0.20 \mathrm{~V} / \mathrm{cm}$ lower in the peritumoral border zone for the patient shown in Fig. 7 when the $\mathrm{dM}$ anisotropic conductivity tensor is used compared to isotropic scalar conductivity assignment. In WM, GM, and tumor tissue, no significant differences in the central tendencies of the field estimates were observed although the choice of conductivity model did affect the topographical distribution of the field in all tissues. Specifically, the interquartile range of the paired difference in field estimates for all elements was $>0.40 \mathrm{~V} / \mathrm{cm}$ for all tissues. The fact that the peritumoral region is more sensitive to the chosen conductivity model, likely indicates that standardized conductivity values for GM and WM do not accurately represent the tissue properties in this region due to the presence of edema and tumor infiltration.

Furthermore, a sensitivity analysis has been carried out to determine how uncertainties in the dielectric properties of tissues affect the EF in the tumor [75], [100]. Variations of permittivity values by up to twofold in all of the head tissues lead to changes in the average field intensity of less than $2 \%$ [75]. This is due to the fact that for TTFields settings the current is mainly resistive as opposed to capacitive, i.e., $\sigma \gg \omega \varepsilon_{0} \varepsilon_{r}$ [102]. Furthermore, this result validates the approach of considering a static problem by neglecting the permittivity, as it was done in the patient studies using SimNibs. However, significant differences in the induced EF intensities have been observed when conductivity values of both pathological and healthy tissues are varied by up to 2.5 -fold [75]. The percentage difference between the highest and the lowest average field intensity in the brain changes up to
$42 \%$. Within the tumor the differences in field intensities can be as large as $68 \%$. The tissues whose conductivity has the greatest effect on the average EF in the tumor are the tumor itself, the skull, and the scalp [75].

The influence of tumor composition on the field intensity was also investigated [79], [100], [103]. The virtual cystic tumors discussed above (with a necrotic core) were replaced with a solid enhancing tumor mass [79], [103]. Although the average field intensity in the active part of the tumor is similar in both cases, the EF distribution is relatively uniform in the solid tumor, but shows hot spots and less exposed areas within the active region, when a necrotic core is introduced.

A consecutive study investigated the influence of tumor size, shape, and position on EF intensity [39]. A virtual tumor with the same shape and size as the tumor discussed above was placed at different location within the brain. Two additional tumors with irregular shape and larger sizes were placed in the left hemisphere (see Fig. 8). The computational results show that tumor shape and size, up to $26.6 \mathrm{~cm}^{3}$ total volume, did not have a noticeable impact on the average EF intensity in the tumor, owing primarily to the large size of the individual arrays. Simulations revealed that the induced average EF intensity value remained above $1 \mathrm{~V} / \mathrm{cm}$ for all tumors irrespective of the direction from which TTFields was delivered. However, visual examination of the field distribution suggested that the default position of the arrays tested in these simulations might not deliver optimal field intensities to all tumors. Tumors in the anterior region are not well covered by the default LR array placed above the ears, although their proximity to the frontal AP patch has been shown to induce high field intensities (see Fig. 8). For superficial tumors, the patches are too low to induce optimal field delivery. The 
study therefore investigated how adapting the transducer arrays positions to specific tumor location influenced field intensity. Indeed, for all tumor locations, moving the patches closer to the region containing the tumor increased the induced average EF intensity in the tumor (see Fig. 8). Details can be found in [39].

Since there exist models of two individuals with the same virtual tumor in terms of size, position, and properties, conclusions about patient variability can be drawn [75], [104]. The second model generally showed higher EF intensities, with average values $20 \%$ and $50 \%$ higher for LR and AP configurations, respectively. The two heads have different shape and size (the first model head is bigger), and an exploratory analysis showed that the median thickness of the scalp and skull are 94\% and $75 \%$ smaller, respectively, in the second model. Thickening the skull in the second model and increasing the head size of the second model [39] bring the average EF intensities in the tumor closer to the values observed in the first model. These studies illustrate the large extent to which the individual head shape and size, and the geometry and thickness of the tissue compartments [100] influence TTFields distribution within the head.

3) Discussion and Future Directions: Currently, there are no noninvasive methods to image the induced TTFields in the head during therapy. Only one intracranial measurement of TTFields intensity has been performed on a human subject. This measurement showed that TTFields intensity within the center of the brain ranges between 1 and $2 \mathrm{~V} / \mathrm{cm}$, and is within $10 \%$ of the predicted values [6]. The development of realistic human head models has allowed detailed description of EF distribution in the head following Optune treatment. This is an important step toward understanding the basic features of the induced field, which are not entirely intuitive. The induced field is highly nonuniform and depends on the complex tissue interfaces and their dielectric property distributions. This results in regions of high field intensity not only close to active transducers but also in deep areas of the brain.

Realistic computational models have also enabled quantification of the EF intensity delivered to the tumor, and show that TTFields intensity in large regions of tumors exceeds the therapeutic threshold of $1 \mathrm{~V} / \mathrm{cm}$, irrespective of head geometry, tumor location and size, and array configuration. The field estimates are influenced by model uncertainties, particularly by uncertainties of the chosen ohmic tissue conductivities. The conductivity is frequency dependent [105] and the intermediate frequency range is least studied and experimental results often cover the low-frequency or higher frequency regimes. In addition, the values reported in the literature possess a substantial spread. Given that the computational study employing a virtual tumor model demonstrated a clear sensitivity of the calculated fields on the chosen tissue conductivities, specific measurements would be highly desirable and would certainly provide an opportunity to improve the accuracy and precision of the model results. This not only relates to the conductivity of the main tissues (skin, skull, CSF, brain gray and white matter), but also to the conductivity of the tumor tissue, which might possess a wide interindividual spread due to differences in tumor type and "stage of development." The latter notion is supported by the documented interindividual variation in apparent diffusion coefficient (ADC) in the tumor region and also the established correlation between ADC values and glioma grade [106].

Virtual tumor models have the advantage of being versatile and able to simulate virtually every situation in terms of tumor location, shape, and size, as well as head shape and size. The influence of each of these parameters can be studied systematically. One study investigated adapting transducer arrays to specific tumor locations, to clarify how beneficial the treatment planning with NovoTAL might be. The modeling results confirmed that the EF intensities can be significantly increased when a patient-specific array layout is used. However, only one subject was tested with virtual tumors and another study already predicted differences in the induced field for two different persons. Computational modeling has the potential to identify the properties of the head that result in these differences. One small study started to investigate the effect of different head size and thicknesses of outer tissue layers. It would be possible to deform the virtual tumor model into different shapes and scale tissue to produce a more individual model [107]. However, these techniques might underestimate altered anatomy due to disease (edema, midline shifts, etc.) in a real patient.

Thus, patient head models, which are created by directly segmenting patient MRI datasets, are a highly important addition to the computational simulation of TTFields application in clinical settings. Such patient-specific models have recently been applied in a study aiming to investigate the potential benefit of combining TTFields with surgical craniectomy [82]. A clinical phase I trial testing the safety and feasibility of this procedure was recently launched (NCT02893137). This example highlights how computational modeling can facilitate translational research.

Despite the considerable potential of patient-specific models, further investigations are required to firmly validate the technique. An important aspect will be the further validation of the dMRI approach for conductivity estimation on a larger number of patients, potentially by correlating dMRI estimates of conductivity to stereotactic in vivo measurements from the same patient [82].

Furthermore, ongoing studies by the authors aim to characterize the importance of tumor locations and morphological characteristics, such as size, shape, mean conductivity distribution, and fractional conductivity anisotropy, on the distribution of the induced field [82]. Such an investigation would be an important addition to the studies performed with the virtual head models, because of the valuable insight on the tumor's heterogeneous environment and its influence on the induced field. A recent study presented a first attempt to create simpler head models that can provide accurate results for calculating the EF distribution for the application of TTFields [108]. However, future feasibility studies based on a number of datasets should address the performance of this simplified model.

Currently, the frameworks available for creating patientspecific models are cumbersome and time-consuming [82]. This is primarily because algorithms for automatic tumor segmentation are inaccurate. Hence, considerable manual intervention is required to obtain models that accurately represent patients. For successful clinical implementation of future modeling 
technologies, the time required to create models and run simulation should be minimized. Thus, an effort should be made to improve segmentation and model creation algorithms to obtain the goal of rapid patient-specific modeling; a parallel investigation into how much complexity is needed in a model to produce reliable predictions of the EF distribution should also be performed.

Clinical investigations have shown a favorable safety profile of TTFields and Optune in particular. Already the virtual head models were used to evaluate specific absorption rate (SAR) values in the scalp, skull, and brain tissues [73], [75]. In the patient head models, peak SAR values were increased by craniectomy but still within the range of median SAR values previously reported [82]. It was concluded that the treatment would not impose additional risk of overheating or damage of healthy tissue [82]. Apart from investigating induced SAR values, these modeling studies could also be adapted to specifically study the heat distribution in the head from Optune treatment.

There are many avenues through which computational head models could be used to address clinical questions. The tools described here could be employed to conduct a retrospective outcome analysis in which patient-specific models are created and the connection between disease progression and field distribution could be investigated. Computational models combined with a deep understanding on how field distribution influences disease progression could then be used for adaptive treatment planning. Realistic head models of a patient could be created periodically during the course of treatment, reflecting dynamic changes in tumor morphology and tissue properties [82]. These models could be combined with simulations and an understanding of field intensity influences disease progression to optimize TTFields delivery in a dynamic manner throughout the course of treatment.

\section{SUMmARY AND CONCLUSION}

In this review, we showed how modeling at different scales can be employed to study TTFields application. At a cellular scale, simulation studies can be used to strengthen physical understanding and basic knowledge of cell-EF interaction. With the creation of realistic human head model, it is possible to investigate TTFields in clinical practice by analyzing induced EF distributions in the patient's brain and tumor during Optune treatment. Important insights can be obtained from these calculations, such as retrospective analysis of treatment outcome as well as prospective personalized treatment planning. In conclusion, TTFields is yet another application, where computational modeling acts as an integral part for further development and efficacy improvement of a therapy. Clinical and engineering communities will greatly benefit by collaborating and drawing from common resources and knowledge.

\section{REFERENCES}

[1] J. Malmivuo and R. Plonsey, Bioelectromagnetism. New York, NY, USA: Oxford Univ. Press, 1995.

[2] G. H. Markx, "The use of electric fields in tissue engineering: A review," Organogenesis, vol. 4, no. 1, pp. 11-17, Jan. 2008.
[3] K. F. Chu and D. E. Dupuy, "Thermal ablation of tumours: Biological mechanisms and advances in therapy," Nature Rev. Cancer, vol. 14, pp. 199-208, 2014.

[4] A. M. Davies, U. Weinberg, and Y. Palti, "Tumor treating fields: A new frontier in cancer therapy," Ann. New York Acad. Sci., vol. 1291, pp. 8695, Jul. 2013.

[5] E. D. Kirson et al., "Disruption of cancer cell replication by alternating electric fields," Cancer Res., vol. 64, no. 9, pp. 3288-3295, May 2004.

[6] E. D. Kirson et al., "Alternating electric fields arrest cell proliferation in animal tumor models and human brain tumors," Proc. Nat. Acad. Sci. USA, vol. 104, no. 24, pp. 10152-10157, Jun. 2007.

[7] M. Giladi et al., "Mitotic disruption and reduced clonogenicity of pancreatic cancer cells in vitro and in vivo by tumor treating fields," Pancreatology, vol. 14, no. 1, pp. 54-63, 2014.

[8] A. Pavesi et al., "Engineering a 3D microfluidic culture platform for tumor-treating field application," Sci. Rep., vol. 6, 2016, Art. no. 26587.

[9] E. H. Kim, H. S. Song, S. H. Yoo, and M. Yoon, "Tumor treating fields inhibit glioblastoma cell migration, invasion and angiogenesis," Oncotarget, vol. 7, no. 40, pp. 65125-36, 2016.

[10] E. H. Kim, Y. J. Kim, H. S. Song, Y. K. Jeong, and J. Y. Lee, "Biological effect of an alternating electric field on cell proliferation and synergistic antimitotic effect in combination with ionizing radiation," Oncotarget vol. 7, no. 38, pp. 62267-62279, 2016.

[11] T. Voloshin et al., "Alternating electric fields (TTFields) in combination with paclitaxel are therapeutically effective against ovarian cancer cells in vitro and in vivo," Int. J. Cancer, vol. 139, no. 12, pp. 2850-2858, 2016.

[12] M. Giladi et al., "Mitotic spindle disruption by alternating electric fields leads to improper chromosome segregation and mitotic catastrophe in cancer," Sci. Rep., vol. 5, 2015, Art. no. 18046.

[13] N. Gera, A. Yang, T. S. Holtzman, S. X. Lee, and E. T. Wong, "Tumor treating fields perturb the localization of septins and cause aberrant mitotic exit," PLoS One, vol. 10, no. 5, pp. 1-20, 2015.

[14] E. D. Kirson et al., "Chemotherapeutic treatment efficacy and sensitivity are increased by adjuvant alternating electric fields (TTFields)," $B M C$ Med. Phys., vol. 9, no. 1, pp. 1-13, 2009.

[15] R. S. Schneiderman, E. Shmueli, E. D. Kirson, and Y. Palti, "TTFields alone and in combination with chemotherapeutic agents effectively reduce the viability of MDR cell sub-lines that over-express ABC transporters," BMC Cancer, vol. 10, May 2010, Art. ID 229.

[16] P. A. Clark et al., "The effects of tumor treating fields and temozolomide in MGMT expressing and non-expressing patient-derived glioblastoma cells," J. Clin. Neurosci., vol. 36, pp. 120-124, 2017.

[17] N. K. Karanam et al., "Tumor-treating fields elicit a conditional vulnerability to ionizing radiation via the downregulation of BRCA1 signaling and reduced DNA double-strand break repair capacity in non-small cell lung cancer cell lines," Cell Death Dis., vol. 8, 2017, Art. no. e2711.

[18] A. Mershin, A. Kolomenski, H. Schuessler, and D. Nanopoulos, "Tubulin dipole moment, dielectric constant and quantum behavior: Computer simulations, experimental results and suggestions," Biosystems., vol. 77, nos. 1-3, pp. 73-85, Nov. 2004.

[19] H. A. Pohl, Dielectrophoresis. Cambridge, U.K.: Cambridge Univ. Press, 1978.

[20] J. A. Tuszyński, C. Wenger, D. Friesen, and J. Preto, "An overview of sub-cellular mechanisms involved in the action of TTFields," Int. $J$. Environ. Res. Public Health, vol. 13, no. 11, 2016, Art. no. E1128.

[21] W. Wick, "TTFields: Where does all the skepticism come from?," NeuroOncology, vol. 18, no. 3, pp. 303-305, 2016.

[22] E. D. Kirson et al., "Alternating electric fields (TTFields) inhibit metastatic spread of solid tumors to the lungs," Clin. Exp. Metastasis, vol. 26, no. 7, pp. 633-640, 2009.

[23] M. Giladi et al., "Alternating electric fields (tumor-treating fields therapy) can improve chemotherapy treatment efficacy in non-small cell lung cancer both in vitro and in vivo," Seminars Oncol., vol. 41, no. 5, pp. S35S41, 2014.

[24] M. Salzberg, E. Kirson, Y. Palti, and C. Rochlitz, "A pilot study with very low-intensity, intermediate-frequency electric fields in patients with locally advanced and/or metastatic solid tumors," Onkologie, vol. 31, no. 7, pp. 362-365, 2008.

[25] A. M. Rulseh et al., "Long-term survival of patients suffering from glioblastoma multiforme treated with tumor-treating fields," World $J$. Surg. Oncol., vol. 10, Oct. 2012, Art. ID 220.

[26] R. Stupp et al., "NovoTTF-100A versus physician's choice chemotherapy in recurrent glioblastoma: A randomised phase III trial of a novel treatment modality," Eur. J. Cancer, vol. 48, no. 14, pp. 2192-2202, Sep. 2012. 
[27] R. Stupp et al., "Maintenance therapy with tumor-treating fields plus temozolomide vs temozolomide alone for glioblastoma. A randomized clinical trial," JAMA, vol. 314, no. 23, pp. 2535-2543, 2015.

[28] R. Stupp et al., "NT-40 Interim analysis of the EF-14 trial: A prospective, multi-center trial of NovoTTF-100A together with temozolomide compared to temozolomide alone in patients with newly diagnosed GBM,' Neuro-Oncology, vol. 16, 2014, Art. no. v167.

[29] R. Stupp et al., "Prospective, multi-phase III trial of tumor treating fields together with Temozolomide comparted to Temozolomide alone in patients with newly diagnosed glioblastoma," Neuro-Oncology, vol. 182016, Art. no. LTBK-01.

[30] U. Weinberg, O. Farber, M. Giladi, Z. Bomzon, and E. D. Kirson, "194TiP: LUNAR: Phase III study of front-line chemotherapy with TTFields for advanced squamous NSCLC," J. Thoracic Oncol., vol. 11, 2016, Art. no. S141.

[31] M. Pless, C. Droege, R. von Moos, M. Salzberg, and D. Betticher, "A phase I/II trial of tumor treating fields (TTFields) therapy in combination with pemetrexed for advanced non-small cell lung cancer," Lung Cancer, vol. 81, no. 3, pp. 445-450, 2013.

[32] F. Rivera, J. Gallego, C. Guillen, M. Benavides, J. A. Lopez-Martin, and M. Kueng, "PANOVA: A pilot study of TTFields concomitant with gemcitabine for front-line therapy of advanced pancreatic adenocarcinoma," Ann. Oncol., vol. 27, pp. 1-85, 2016.

[33] E. Fonkem and E. T. Wong, "NovoTTF-100A: a new treatment modality for recurrent glioblastoma," Expert Rev. Neurotherapeutics, vol. 12, pp. 895-899, 2012

[34] M. M. Mrugala et al., "Clinical practice experience with NovoTTF100ATM system for glioblastoma: The patient registry dataset (PRiDe),' Seminars Oncol., vol. 41 no. 5, pp. S4-S13, Oct. 2014.

[35] Z. Bomzon et al., "Using computational phantoms to improve delivery of tumor treating fields (TTFields) to patients," in Proc. Conf. Proc. IEEE Eng. Med. Biol. Soc., 2016, pp. 6461-6464.

[36] A. Chaudhry et al., "NovoTTF-100A System (Tumor Treating Fields) transducer array layout planning for glioblastoma: A NovoTAL system user study," World J. Surg. Oncol., vol. 13, Nov. 2015, Art. ID 316.

[37] J. Trusheim et al., "A state-of-the-art review and guidelines for tumor treating fields treatment planning and patient follow-up in glioblastoma,' CNS Oncol., vol. 6, pp. 29-43, Sep. 2016.

[38] C. Wenger, R. Salvador, P. J. Basser, and P. C. Miranda, "Increasing tumor treating fields (TTFields) efficacy in GBM patients through optimization of transducer array configuration," Neuro-Oncology, vol. 17, Nov. 2015, Art. ID v128.

[39] C. Wenger, R. Salvador, P. J. Basser, and P. C. Miranda, "Improving TTFields treatment efficacy in patients with glioblastoma using personalized array layouts," Int. J. Radiative Oncol., vol. 94, no. 5, pp. 1137 $1143,2016$.

[40] P. Y. Wen and D. A. Reardon, "Neuro-oncology in 2015: Progress in glioma diagnosis, classification and treatment," Nat. Rev. Neurol., vol. 12, pp. 69-70, 2016

[41] D. N. Louis et al., "The 2016 world health organization classification of tumors of the central nervous system: A summary," Acta Neuropathol., vol. 131 , no. 6, pp. 803-820, 2016.

[42] H. A. Haus and J. R. Melcher, Electromagnetic Fields and Energy. Cambridge, MA, USA: Massachusetts Inst. Technol., MIT OpenCourseWare.

[43] S. Watkins and H. Sontheimer, "Unique biology of gliomas: Challenges and opportunities," Trends Neurosci., vol. 35, no. 9, pp. 546-556, 2013.

[44] C. Wenger, P. C. Miranda, R. Salvador, and P. J. Basser, "Investigating the mechanisms of action of tumor treating fields: A computational modeling study," Neuro-Oncology, vol. 16, 2014, Art. no. v216.

[45] C. Wenger, M. Giladi, Z. Bomzon, R. Salvador, P. J. Basser, and P. C. Miranda, "Modeling tumor treating fields (TTFields) application in single cells during metaphase and telophase," in Proc. Conf. Proc. IEEE Eng. Med. Biol. Soc., 2015, pp. 6892-6895.

[46] C. Wenger and P. C. Miranda, "Biophysical effects of alternating electric fields," in Alternating Electric Fields Therapy in Oncology. New York, NY, USA: Springer, 2016.

[47] D. Stewart, T. R. Gowrishankar, K. C. Smith, and J. C. Weaver, "Cylindrical cell membranes in uniform applied electric fields: Validation of a transport lattice method," IEEE Trans. Biomed. Eng., vol. 52, no. 10, pp. 1643-53, Oct. 2005.

[48] T. R. Gowrishankar and J. C. Weaver, "An approach to electrical modeling of single and multiple cells," Proc. Nat. Acad. Sci. USA., vol. 100, no. 6, pp. 3203-3208, Mar. 2003.
[49] T. R. Gowrishankar, D. A. Stewart, and J. C. Weaver, "Model of a confined spherical cell in uniform and heterogeneous applied electric fields," Bioelectrochemistry, vol. 68, no. 2, pp. 181-190, May 2006.

[50] V. Vajrala, J. R. Claycomb, H. Sanabria, and J. H. Miller, "Effects of oscillatory electric fields on internal membranes: An analytical model," Biophys. J., vol. 94, no. 6, pp. 2043-2052, Mar. 2008.

[51] P. K. Tiwari, S. K. Kang, G. J. Kim, J. Choi, A.-A. H. Mohamed, and J. K. Lee, "Modeling of nanoparticle-mediated electric field enhancement inside biological cells exposed to AC electric fields," Jpn. J. Appl. Phys., vol. 48, no. 8, Aug. 2009, Art. no. 087001.

[52] T. Kotnik, F. Bobanović, and D. Miklavčič, "Sensitivity of transmembrane voltage induced by applied fields - a theoretical analysis," Bioelectrochem. Bioenergetics, vol. 43, no. 43, pp. 285-291, 1997.

[53] T. Kotnik and D. Miklavčič, "Second-Order model of membrane electric field induced by alternating external electric fields," IEEE Trans. Biomed. Eng., vol. 47, no. 8, pp. 1074-1081, Aug. 2000.

[54] T. Kotnik and D. Miklavčič, "Theoretical evaluation of voltage inducement on internal membranes of biological cells exposed to electric fields," Biophys. J., vol. 90, no. 2, pp. 480-491, Jan. 2006.

[55] H. Ye, M. Cotic, E. E. Kang, M. G. Fehlings, and P. L. Carlen, "Transmembrane potential induced on the internal organelle by a time-varying magnetic field: A model study," J. Neuroeng. Rehabil., vol. 7, Feb. 2010, Art. ID 12.

[56] H. Ye, M. Cotic, M. G. Fehlings, and P. L. Carlen, "Influence of cellular properties on the electric field distribution around a single cell," Prog. Electromagn. Res. B, vol. 39, pp. 141-161, 2012.

[57] T. Sun, H. Morgan, and N. Green, "Analytical solutions of ac electrokinetics in interdigitated electrode arrays: Electric field, dielectrophoretic and traveling-wave dielectrophoretic forces," Phys. Rev. E, vol. 76, no. 4, Oct. 2007, Art. no. 046610

[58] T. B. Jones, "Basic theory of dielectrophoresis and electrorotation," IEEE Eng. Med. Biol. Mag., vol. 22, no. 6, pp. 33-42, Nov./Dec. 2003.

[59] C. Vaillier, T. Honegger, F. Kermarrec, X. Gidrol, and D Peyrade, "Labelfree electric monitoring of human cancer cells as a potential diagnostic tool," Anal. Chem., vol. 88, no. 18, pp. 9022-9028, 2016.

[60] M. Giladi et al., "ET-029. Overcoming cell size escape from tumor treating fields using a varying frequency treatment paradigm in-vitro," Neuro-Oncology, vol. 15, pp. iii37-iii61, 2013.

[61] M. Cooper, 'Coherent polarization waves in cell division and cancer,' Collective Phenom., vol. 3, pp. 273-288, 1981.

[62] H. A. Pohl, T. Braden, S. Robinson, J. Piclardi, and D. G. Pohl, "Life cycle alterations of the micro-dielectrophoretic effects of cell," J. Biol. Phys., vol. 9, no. 3, pp. 133-154, 1981.

[63] H. A. Pohl, "Oscillating fields about growing cells," Int. J. Quantum Chem., vol. 18, pp. 411-431, 1980.

[64] F. Jelínek, J. Pokorný, J. Saroch, V. Trkal, J. Hasek, and B. Palán, "Microelectronic sensors for measurement of electromagnetic fields of living cells and experimental results," Bioelectrochem. Bioenergetics, vol. 48, no. 2, pp. 261-266, 1999.

[65] L. J. Gagliardi, "Electrostatic force generation in chromosome motions during mitosis," J. Electrostatics, vol. 63, nos. 3/4, pp. 309-327, 2005.

[66] L. J. Gagliardi, "Electrostatic force in prometaphase, metaphase, and anaphase-A chromosome motions," Phys. Rev. E, vol. 66, no. 1, pt. 1, Jul. 2002, Art. no. 011901.

[67] L. J. Gagliardi, "Microscale electrostatics in mitosis," J. Electrostatics, vol. 54, nos. 3/4, pp. 219-232, 2002.

[68] D. Havelka, M. Cifra, O. Kučera, J. Pokorný, and J. Vrba, "Highfrequency electric field and radiation characteristics of cellular microtubule network," J. Theor. Biol., vol. 286, no. 1, pp. 31-40, Oct. 2011.

[69] M. Cifra, D. Havelka, and O. Kučera, "Biophysical role of oscillatory electric field generated by undamped microtubule vibrations," in Proc. 6th Int. Workshop Biol. Effects Electromagn. Fields, Bogazici University, Istanbul, 2010, pp. 1-5.

[70] D. Havelka, O. Kučera, M. A. Deriu, and M. Cifra, "Electro-Acoustic behavior of the mitotic spindle: A semi-classical coarse-grained model," PLoS One, vol. 9, no. 1, Jan. 2014, Art. no. e86501.

[71] K. M. Tyner, R. Kopelman, and M. A. Philbert, "'Nanosized voltmeter' enables cellular-wide electric field mapping," Biophys. J., vol. 93, no. 4 pp. 1163-1174, 2007.

[72] C. Wenger, P. C. Miranda, A. Mekonnen, R. Salvador, and P. J. Basser, "TM-028. Electric fields for the treatment of glioblastomas: A modeling study," Neuro-Oncology, vol. 15, pp. iii235-iii241, 2013.

[73] P. C. Miranda, A. Mekonnen, R. Salvador, and P. J. Basser, "Predicting the electric field distribution in the brain for the treatment of glioblastoma," Phys. Med. Biol., vol. 59, no. 15, pp. 4137-4147, 2014 
[74] D. W. Shattuck and R. M. Leahy, "BrainSuite: An automated cortical surface identification tool," Med. Image Anal., vol. 6, no. 2, pp. 129-142, Jun. 2002.

[75] C. Wenger, R. Salvador, P. J. Basser, and P. C. Miranda, "The electric field distribution in the brain during TTFields therapy and its dependence on tissue dielectric properties and anatomy: A computational study," Phys. Med. Biol., vol. 60, no. 18, pp. 7339-7357, 2015.

[76] M. Windhoff, A. Opitz, and A. Thielscher, "Electric field calculations in brain stimulation based on finite elements: An optimized processing pipeline for the generation and usage of accurate individual head models," Hum. Brain Mapping, vol. 34, no. 4, pp. 923-935, Apr. 2013.

[77] B. Fischl, "FreeSurfer," Neuroimage, vol. 62, no. 2, pp. 774-781, Aug. 2012.

[78] M. Jenkinson, C. F. Beckmann, T. E. J. Behrens, M. W. Woolrich, and S. M. Smith, "FSL," Neuroimage, vol. 62, no. 2, pp. 782-790, Aug. 2012.

[79] A. R. Korshoej, F. L. Hansen, A. Thielscher, G. B. Von Oettingen, J. Christian, and S. Hedemann, "Impact of tumor position, conductivity distribution and tissue homogeneity on the distribution of tumor treating fields in a human brain: A computer modeling study," PLoS One, vol. 12, no. 6, 2017, Art. no. e0179214.

[80] E. Lok, V. Hua, and E. T. Wong, "Computed modeling of alternating electric fields therapy for recurrent glioblastoma," Cancer Med., vol. 4, pp. 1697-1699, 2015.

[81] E. T. Wong, E. Lok, and K. D. Swanson, "An evidence-based review of alternating electric fields therapy for malignant gliomas," Curr. Treatment Options Oncol., vol. 16, no. 8, p. 40, 2015.

[82] A. R. Korshoej, G. B. Saturnino, K. Rasmussen, G. von Oettingen, C. J. Hedemann Sørensen, and A. Thielscher, "Enhancing predicted efficacy of tumor treating fields therapy of glioblastoma using targeted surgical craniectomy: A computer modeling study," PLoS One, vol. 11, no. 10, pp. 1-25, 2016.

[83] M. Attene and B. Falcidieno, "ReMESH: An interactive environment to edit and repair triangle meshes," in Proc. Shape Model. Int., 2006, pp. 271-276.

[84] C. Geuzaine and J.-F. Remacle, "Gmsh: A three-dimensional finite element mesh generator with built-in pre- and post-processing facilities," Int. J. Numer. Methods Eng., vol. 79, pp. 1309-1331, 2009.

[85] P. Dular, C. Geuzaine, F. Henrotte, and W. Legros, "A general environment for the treatment of discrete problems and its application to the finite element method,' IEEE Trans. Magn., vol. 34, no. 5, pp. 3395-3398, Sep. 1998.

[86] W. H. Lee, Z. D. Deng, A. F. Laine, S. H. Lisanby, and A. V. Peterchev, "Influence of white matter conductivity anisotropy on electric field strength induced by electroconvulsive therapy," in Proc. Conf. Proc. IEEE Eng. Med. Biol. Soc., 2011, pp. 5473-5476.

[87] S. Shahid, P. Wen, and T. Ahfock, "Numerical investigation of white matter anisotropic conductivity in defining current distribution under tDCS," Comput. Methods Programs Biomed., vol. 109, no. 1, pp. 48-64, Jan. 2013.

[88] S. Shahid, P. Wen, and T. Ahfock, "Assessment of electric field distribution in anisotropic cortical and subcortical regions under the influence of tDCS," Bioelectromagnetics, vol. 57, pp. 41-57, Oct. 2014.

[89] H. S. Suh, W. H. Lee, and T. Kim, "Influence of anisotropic conductivity in the skull and white matter on transcranial direct current stimulation via an anatomically realistic finite element head model," Phys. Med. Biol., vol. 57, no. 21, pp. 6961-6980, 2012.

[90] P. J. Basser, J. Mattiello, and D. LeBihan, "MR Diffusion Tensor Spectroscopy and Imaging,” Biophys. J., vol. 66, pp. 259-267, 1994.

[91] C. Pierpaoli and P. J. Basser, "Toward a quantitative assessment of diffusion anisotropy," Magn. Reson. Med., vol. 36, no. 6, pp. 893-906, 1996.
[92] S. M. Smith et al., "Advances in functional and structural MR image analysis and implementation as FSL," Neuroimage, vol. 23, pp. 208219, 2004.

[93] A. Opitz, M. Windhoff, R. M. Heidemann, R. Turner, and A. Thielscher, "How the brain tissue shapes the electric field induced by transcranial magnetic stimulation," Neuroimage, vol. 58, no. 3, pp. 849-859, Oct. 2011.

[94] D. S. Tuch, V. J. Wedeen, M. Dale, J. S. George, and J. W. Belliveau, "Conductivity tensor mapping of the human brain using diffusion tensor MRI," Proc. Nat. Acad. Sci. USA, vol. 98, no. 20, pp. 11697-701, Sep. 2001.

[95] M. Rullmann, A. Anwander, M. Dannhauser, S. K. Warfield, F. H. Duffy, and C. H. Wolters, "EEG source analysis of epileptiform activity using $1 \mathrm{~mm}$ anisotropic hexahedra finite element head model," Neuroimage, vol. 44, no. 2, pp. 399-410, 2009.

[96] D. Güllmar, J. Haueisen, and J. R. Reichenbach, "Influence of anisotropic electrical conductivity in white matter tissue on the EEG/MEG forward and inverse solution. A high-resolution whole head simulation study," Neuroimage, vol. 51, no. 1, pp. 145-163, May 2010.

[97] J. Latikka, T. Kuurne, and H. Eskola, "Conductivity of living intracranial tissues,” Phys. Med. Biol., vol. 46, no. 6, pp. 1611-1616, 2001.

[98] R. Peloso, D. T. Tuma, and R. K. Jain, "Dielectric properties of solid tumors during normothermia and hyperthermia," IEEE Trans. Biomed. Eng., vol. 31, no. 11, pp. 725-728, Nov. 1984.

[99] Y. Lu, B. Li, J. Xu, and J. Yu, "Dielectric properties of human glioma and surrounding," Int. J. Hyperthermia, vol. 8, no. 6, pp. 755-760, 1992.

[100] E. Lok, P. San, V. Hua, M. Phung, and E. T. Wong, "Analysis of physical characteristics of tumor treating fields for human glioblastoma," Cancer Med., vol. 6, no. 6, pp. 1286-1300, 2017.

[101] P. C. Miranda, M. Hallett, and P. J. Basser, "The electric field induced in the brain by magnetic stimulation: A 3-D finite-element analysis of the effect of tissue heterogeneity and anisotropy," IEEE Trans. Biomed. Eng., vol. 50, no. 9, pp. 1074-85, Sep. 2003.

[102] R. Plonsey and D. B. Heppner, "Considerations of quasi-stationarity in electrophysiological systems," Bull. Math. Biophys., vol. 29, pp. 657-64, 1967.

[103] C. Wenger, P. C. Miranda, R. Salvador, and P. J. Basser, "Alternating electric fields (TTFields) for treating glioblastomas: A modeling study on efficacy," Neuro-Oncology, vol. 16, Sep. 2014, Art. ID ii92.

[104] C. Wenger, R. Salvador, P. Basser, and P. Miranda, "Modeling intersubject differences in tumor treating fields (TTFields) treatment of GBM patients," Int. J. Radiat. Oncol. Biol. Phys., vol. 33, no. 3, p. E71, Nov. 2015.

[105] C. Gabriel, S. Gabriel, and E. Corthout, "The dielectric properties of biological tissues: I. Literature survey," Phys. Med. Biol., vol. 41, no. 11, pp. 2231-2249, Nov. 1996.

[106] Y. Kang et al., "Gliomas: Histogram analysis of apparent diffusion coefficient maps with standard-or high-b -Value diffusion-weighted MR imaging - correlation with tumor grade 1," Radiology, vol. 261, no. 3, pp. 882-890, 2011.

[107] H. S. Hershkovich et al., "First steps to creating a platform for high throughput simulation of TTFields," in Proc. Conf. Proc. IEEE Eng. Med. Biol. Soc., 2016, pp. 2357-2360.

[108] C. Wenger, Z. Bomzon, R. Salvador, P. J. Basser, and P. C. Miranda, "Simplified realistic human head model for simulating Tumor Treating Fields (TTFields)," in Proc. Conf. Proc. IEEE Eng. Med. Biol. Soc., 2016, pp. 5664-5667.

Authors' photographs and biographies not available at the time of publication. 